\title{
Truncated stathmin-2 is a marker of TDP-43 pathology in frontotemporal dementia
}

Mercedes Prudencio, ${ }^{1,2}$ Jack Humphrey,, ${ }^{3,4}$ Sarah Pickles, ${ }^{1,2}$ Anna-Leigh Brown, ${ }^{,}$Sarah E. Hill, ${ }^{6}$ Jennifer M. Kachergus, ${ }^{7}$ J. Shi, ${ }^{7}$ Michael C. Heckman, ${ }^{8}$ Matthew R. Spiegel, ${ }^{8}$ Casey Cook, ${ }^{1,2}$ Yuping Song, ${ }^{1}$ Mei Yue, ${ }^{1}$ Lillian M. Daughrity, ${ }^{1}$ Yari Carlomagno, ${ }^{1,2}$ Karen Jansen-West, ${ }^{1}$ Cristhoper Fernandez de Castro, ${ }^{1}$ Michael DeTure, ${ }^{1,2}$ Shunsuke Koga ${ }^{1,2}$ Ying-Chih Wang, ${ }^{4}$ Prasanth Sivakumar, ${ }^{5}$ Cristian Bodo, ${ }^{5}$ Ana Candalija, ${ }^{9}$ Kevin Talbot, ${ }^{9}$ Bhuvaneish T. Selvaraj, ${ }^{10}$ Karen Burr, ${ }^{10}$ Siddharthan Chandran,,${ }^{10}$ Jia Newcombe, ${ }^{11}$ Tammaryn Lashley, ${ }^{12,13}$ Isabel Hubbard, ${ }^{14}$ Demetra Catalano, ${ }^{14}$ Duyang Kim,,${ }^{14}$ Nadia Propp, ${ }^{14}$ Samantha Fennessey, ${ }^{15}$ NYCC ALS Consortium, ${ }^{16}$ Delphine Fagegaltier, ${ }^{14}$ Hemali Phatnani, ${ }^{14}$ Maria Secrier, ${ }^{17}$ Elizabeth M.C. Fisher, ${ }^{5}$ Björn Oskarsson, ${ }^{18}$ Marka van Blitterswijk, ${ }^{1,2}$ Rosa Rademakers, ${ }^{1,2}$ Neil R. Graff-Radford, ${ }^{18}$ Bradley F. Boeve, ${ }^{19}$ David S. Knopman, ${ }^{19}$ Ronald C. Petersen, ${ }^{19}$ Keith A. Josephs, ${ }^{19}$ E. Aubrey Thompson, ${ }^{7}$ Towfique Raj, ${ }^{3,4}$ Michael Ward, ${ }^{6}$ Dennis W. Dickson, ${ }^{1,2}$ Tania F. Gendron, ${ }^{1,2}$ Pietro Fratta, ${ }^{5}$ and Leonard Petrucelli, ${ }^{1,2}$

'Department of Neuroscience, Mayo Clinic, Jacksonville, Florida, USA. ${ }^{2}$ Neuroscience Graduate Program, Mayo Clinic Graduate School of Biomedical Sciences, Jacksonville, Florida, USA. ${ }^{3}$ Ronald M. Loeb Center for Alzheimer's Disease, Nash Family Department of Neuroscience and Friedman Brain Institute, and ${ }^{2}$ Department of Cenetics and Genomic Sciences, Icahn School of Medicine at Mount Sinai, New York, New York, USA. ${ }^{5}$ Department of Neuromuscular Diseases, UCL Queen Square Institute of Neurology, London, United Kingdom. ${ }^{6}$ National Institute of Neurological Disorders and Stroke (NINDS), NIH, Bethesda, Maryland, USA. 'Department of Cancer Biology, and ${ }^{8}$ Division of Biomedical Statistics and Informatics, Mayo Clinic, Jacksonville, Florida, USA. ${ }^{9}$ Nuffield Department of Clinical Neurosciences, University of Oxford, Oxford, United Kingdom. ${ }^{10}$ UK Dementia Research Institute and Euan MacDonald Centre for Motor Neurone Disease (MND) Research, The University of Edinburgh, United Kingdom. "NeuroResource, Department of Neuroinflammation, ${ }^{12}$ Department of Neurodegenerative Disease, and ${ }^{13}$ Queen Square Brain Bank for Neurological Disorders, Department of Clinical and Movement Neuroscience, UCL Queen Square Institute of Neurology, London, United Kingdom. ${ }^{14}$ Center for Cenomics of Neurodegenerative Disease, and ${ }^{15}$ New York Cenome Center (NYGC), New York, New York, USA. ${ }^{16}$ The NYGC ALS Consortium is detailed in Supplemental Acknowledgments. ${ }^{17}$ University College London Genetics Institute, London, United Kingdom. ${ }^{18}$ Department of Neurology, Mayo Clinic, Jacksonville, Florida, USA. ${ }^{19}$ Department of Neurology, Mayo Clinic, Rochester, Minnesota, USA.

No treatment for frontotemporal dementia (FTD), the second most common type of early-onset dementia, is available, but therapeutics are being investigated to target the 2 main proteins associated with FTD pathological subtypes: TDP-43 (FTLD-TDP) and tau (FTLD-tau). Testing potential therapies in clinical trials is hampered by our inability to distinguish between patients with FTLD-TDP and FTLD-tau. Therefore, we evaluated truncated stathmin-2 (STMN2) as a proxy of TDP-43 pathology, given the reports that TDP-43 dysfunction causes truncated STMN2 accumulation. Truncated STMN2 accumulated in human induced pluripotent stem cell-derived neurons depleted of TDP-43, but not in those with pathogenic TARDBP mutations in the absence of TDP-43 aggregation or loss of nuclear protein. In RNA-Seq analyses of human brain samples from the NYCC ALS cohort, truncated STMN2 RNA was confined to tissues and disease subtypes marked by TDP43 inclusions. Last, we validated that truncated STMN2 RNA was elevated in the frontal cortex of a cohort of patients with FTLD-TDP but not in controls or patients with progressive supranuclear palsy, a type of FTLD-tau. Further, in patients with FTLD-TDP, we observed significant associations of truncated STMN2 RNA with phosphorylated TDP-43 levels and an earlier age of disease onset. Overall, our data uncovered truncated STMN2 as a marker for TDP-43 dysfunction in FTD.

\section{Introduction}

Tar DNA-binding protein 43 (TDP-43) regulates multiple aspects of RNA metabolism, including RNA stabilization, transcription, splicing, and transport (1). TDP-43 binds thousands of RNAs (2,

\section{Related Commentary: p. 5677}

Authorship note: MP, JH, and SP are co-first authors and contributed equally to this work. Conflict of interest: RCP is a consultant for Roche Inc., Merck Inc., Biogen Inc., and Eisai Inc.; is a member of the data and safety monitoring board (DSMB) of Genentech; and has provided educational talks for GE Healthcare. Copyright: ( 2020 , American Society for Clinical Investigation. Submitted: April 30, 2020; Accepted: August 11, 2020; Published: October 19, 2020 Reference information: / Clin Invest. 2020;130(11):6080-6092. https://doi.org/10.1172/JCl139741.
3) and, for particular genes, represses nonconserved cryptic exons in a species- (4) and cell-type-specific manner $(5,6)$. Recently, 2 groups independently found that TDP-43 is required for the correct splicing and expression of stathmin-2 (STMN2) $(7,8)$, a microtubule-associated protein involved in axonal regeneration (9). As shown schematically in Figure 1, when TDP-43 is present and functional, it binds to GU-rich sequences in the first intron of the STMN2 transcript, allowing normal splicing of intron 1 and repressing the usage of an alternative or cryptic polyadenylation site. Reduced TDP-43 binding to STMN2 pre-mRNA, which could occur as a result of TDP-43 nuclear depletion, leads to the inclusion of a region within intron 1 (exon $2 \mathrm{a}$ ) and introduces a premature stop codon and polyadenylation. This causes the accumulation of a truncated variant of STMN2 that lacks exons 2 through 5. 


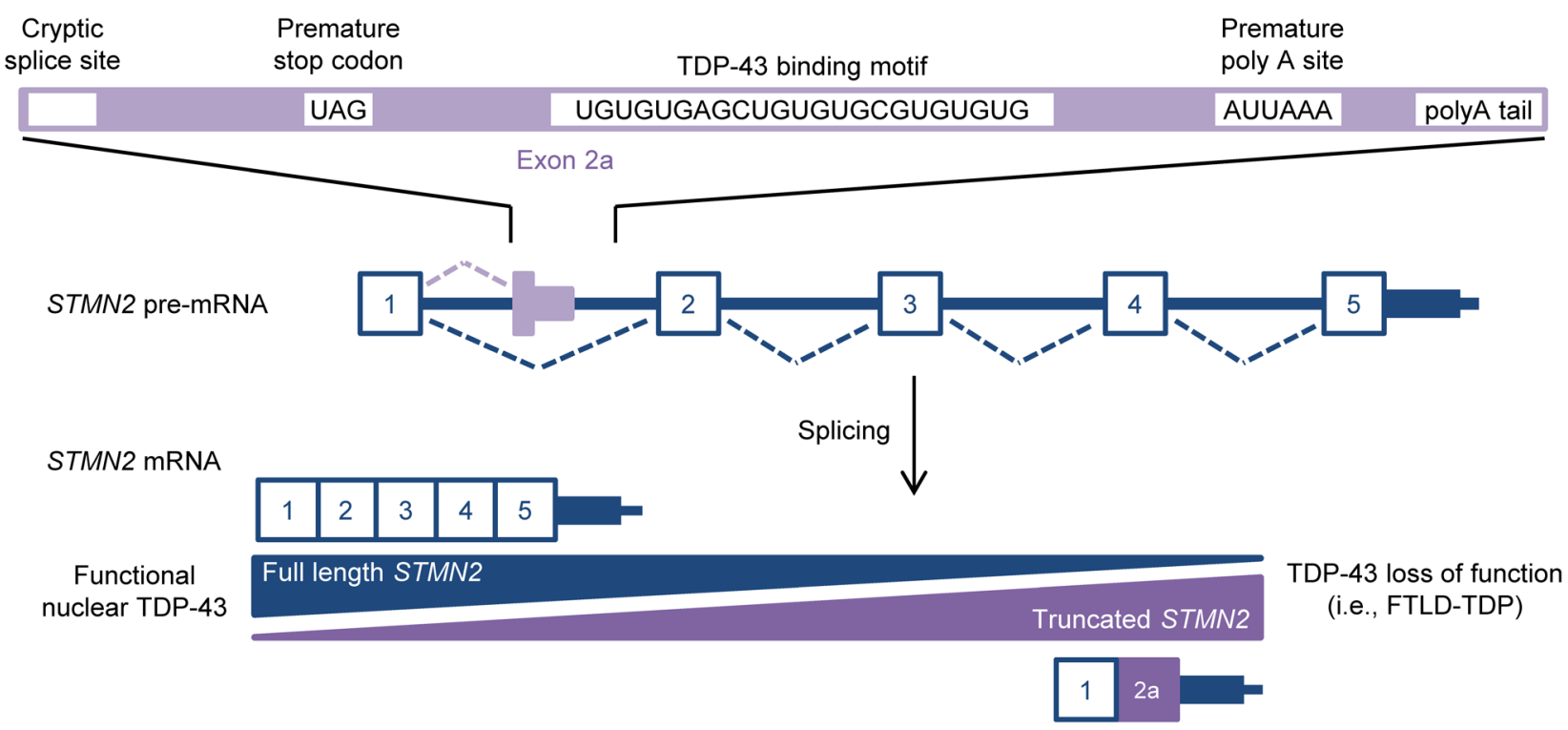

Figure 1. Truncated STMN2 RNA is generated with loss of nuclear TDP-43. Schematic of TDP-43-regulated STMN2 splicing. When TDP-43 is present and functional, it binds to GU-rich sequences in the first intron of the STMN2 transcript and allows normal splicing of intron 1. Reduced TDP-43 binding to STMN2 RNA, from TDP-43 aggregation or depletion from the nucleus, leads to mis-splicing of STMN2 RNA, resulting in the inclusion of a novel exon encoded within intron 1 (termed exon 2a) and containing an alternative or cryptic polyadenylation site. The production of this alternative variant of STMN2, which lacks exons 2 through 5 (referred to as truncated STMN2), is at the expense of full-length STMN2, which is reduced upon TDP-43 downregulation.

Neuronal and glial inclusions of TDP-43 are a pathological hallmark of the majority ( $97 \%$ ) of patients with ALS and of approximately $50 \%$ of cases with the pathological subtype of frontotemporal dementia (FTD), known as frontotemporal lobar degeneration-TDP (FTLD-TDP) (1). ALS is a motor neuron disease (MND), whereas patients with FTD present clinically with 1 or more of the FTD syndromes characterized by behavior, executive function, language, and motor impairments (10). TDP-43 abnormalities are proposed to play a direct role in these diseases, since mutations in TARDBP, the gene that encodes TDP-43, were discovered in sporadic and familial ALS and FTD $(11,12)$. It is also notable that TDP- 43 morphology and distribution are associated with clinical phenotypes and neurodegeneration in key anatomical regions, such as frontal and temporal cortices in FTLD-TDP (13). Cells with TDP-43 pathology feature a redistribution of TDP-43 from the nucleus to the cytoplasm, where TDP- 43 is ubiquitinated, hyperphosphorylated, and cleaved into C-terminal fragments (14). Patients with FTLD-TDP can be further classified into different pathological subtypes on the basis of the morphology and distribution of the TDP-43 inclusions observed $(15,16)$. These can take the form of neuronal cytoplasmic inclusions, dystrophic neurites, neuronal intranuclear inclusions, glial inclusions, perivascular inclusions, and fine neurites in the hippocampus (14, 17-20). At present, 5 subtypes of FTLD-TDP (A-E) have been identified $(15,20-23)$, with types A-C accounting for more than $95 \%$ of all FTLD-TDP cases (24). FTLD-TDP type A, the most common subtype, is characterized by the presence of all the inclusion types mentioned above, whereas types B-E are characterized by the presence of a predominant inclusion type $(19,22)$. Furthermore, some FTLD-TDP subtypes are associated with particular mutations $(20,25,26)$.
While approximately $50 \%$ of patients with an FTD syndrome have underlying TDP-43 pathology, such pathology can, at present, only be definitively confirmed at autopsy. Given the efforts to develop therapies specific to FTLD pathological subtypes (i.e., FTD with TDP- 43 vs. tau pathology), there is a clear need for biomarkers that discriminate between them. Further, biomarkers for diagnosis or prognosis or that can monitor TDP-43 activity in response to TDP-43-tailored therapies are also sorely lacking. Although TDP-43 represents an attractive biomarker candidate to fulfill these needs, efforts to develop sufficiently sensitive immunoassays for the detection of TDP-43 or its pathological forms in biofluids and that reliably differentiate between individuals with and without TDP- 43 pathology have proven disappointing (27). However, nuclear clearance of TDP- 43 , in the absence of TDP- 43 inclusions, has been associated with neuronal atrophy in patients with FTLD-TDP or ALS-TDP (28), suggesting that early mis-splicing events due to TDP- 43 loss of function may represent a robust and early disease biomarker.

We thus propose that the mis-splicing of STMN2 may provide a novel strategy for the indirect assessment of TDP- 43 dysfunction. To test this hypothesis, we first validated the STMN2 splicing event in induced pluripotent stem cell-derived (iPSC-derived) cortical and motor neurons that were TDP- 43 depleted. Then, we evaluated whether the truncated STMN2 isoform accumulates in diseases and tissues characterized by TDP- 43 pathology using data sets from RNA-Seq studies of brain tissues from a large cohort of control subjects and of patients with FTD or ALS with or without TDP-43 pathology. Furthermore, we measured truncated and full-length STMN2 RNA levels in postmortem medial frontal cortex tissue from FTLD-TDP, progressive nuclear palsy (PSP), and pathologically normal control brains. PSP was selected as the disease control, given that TDP-43 pathology is not found in the frontal cortex of these patients with FTLD-tau (29). We addition- 
ally evaluated whether truncated STMN2 levels in the medial frontal cortex discriminated between patients with FTLD-TDP and control subjects and assessed associations of truncated SMTN2 with phosphorylated TDP-43 (p-TDP-43) and clinical features.

\section{Results}

TDP-43 depletion in human iPSC-derived neurons leads to aberrant STMN2 splicing. To confirm recent reports that correct STMN2 splicing requires TDP-43 $(7,8)$, we used clustered regularly interspaced short palindromic repeat inactivation (CRISPRi) to knock down TDP43 expression in human iPSC-derived cortical neurons that constitutively express nuclease deactivated Cas9 (dCas9) fused to blue fluorescent protein (BFP) and the transcriptional repressor Krüppel-associated box (KRAB) (dCas9-BFP-KRAB) (30). To achieve this, cells were transduced with a lentivirus expressing either an sgRNA against the transcriptional start site of TARDBP (TDP-43 KD), or a control sgRNA predicted not to bind anywhere in the human genome (controls). We confirmed silencing of TARDBP RNA by quantitative reverse transcription PCR (qRT-PCR) and RNA-Seq (data set labeled "a"), which showed TDP-43-KD efficiencies of approximately $50 \%$ (47.4\% for qRT-PCR, 63.9\% for RNA-Seq) (Figure 2A). Reduced TARDBP levels resulted in significant reductions of full-length STMN2 RNA (56.7\% for qRT-PCR, 65.5\% for RNA-Seq) (Figure 2B) and a significant increase in truncated STMN2 RNA (qRT-PCR, 32.3fold increase) (Figure 2C) and RNA-Seq (Figure 2D, data set labeled "a") compared with levels in neurons expressing control sgRNA. In parallel, we analyzed published RNA-Seq data from Klim et al. on iPSC-derived motor neurons in which TARDBP was knocked down (7). Knockdown of TARDBP (Supplemental Figure 1A; supplemental material available online with this article; https://doi.org/10.1172/ JCI139741DS1) was confirmed in the Klim data set (data set labeled "b") and, as expected, was accompanied by a reduction in full-length STMN2 RNA levels (Supplemental Figure 1B) and an increase in truncated STMN2 RNA levels (Figure 2D, data set labeled "b").

We also investigated whether disease-causing mutations of $T A R D B P$, in the absence of TDP-43 protein mislocalization or a reduction of nuclear levels, are sufficient to impact STMN2 splicing. We evaluated truncated STMN2 expression in RNA-Seq data from 2 independent studies that used iPSC-derived motor neurons from patients carrying ALS pathogenic TARDBP mutations and from control subjects (Supplemental Table 1). In contrast to neurons in which TARDBP was downregulated, we observed no increase in truncated STMN2 in motor neurons with TARDBP mutations (Figure 2D, data sets labeled "c" and "d"). This observation was consistent with the lack of change in TDP-43 localization (Figure 2E), TARDBP RNA levels (Supplemental Figure 2), and TDP-43 protein levels (Supplemental Figure 3) in TARDBP-mutant motor neurons. Overall, these data suggest that truncated STMN2 accumulation in iPSC-derived neurons results from a loss of TDP43 function due to TDP-43 depletion and not from splicing function changes arising from mutations in TARDBP (31). The redistribution of TDP-43 from the nucleus and its sequestration into cytoplasmic inclusions, a pathological hallmark of FTD and ALS, with and without a genetic link to TARDBP, are consistent with loss-of-function mechanisms that would lead to STMN2 mis-splicing and an upregulation of truncated STMN2 expression and prompted us to further examine this in human tissue from FTD and ALS patients.
Analyses of bulk brain tissue RNA-Seq data uncover tissue-specific truncated STMN2 RNA accumulation in diseases characterized by TDP-43 pathology. We quantified the expression of full-length and truncated STMN2 in the NYGC ALS Consortium RNA-Seq data set across multiple neuroanatomical regions and diseases. As of March 2020, we were able to examine 11 tissues, with 1659 RNA-Seq samples from 439 donors matching established criteria for inclusion (see Methods). Donors were diagnosed as non-neurological disease controls or as having FTD, ALS, FTD with ALS (ALS-FTLD), or ALS with suspected Alzheimer's disease (ALS$\mathrm{AD})$. Patients with FTD were classified on the basis of their neuropathological diagnosis: FTLD-TDP, FTLD-tau, or FTLD with mutations in FUS (FTLD-FUS). ALS samples were divided into those with a SOD1 mutation (ALS-SOD1) versus those without a SOD1 mutation and thus assumed to have TDP-43 pathology (ALS-TDP). However, pathology confirming the presence of TDP-43 mislocalization in patients with ALS-TDP was not systematically reported (see Methods).

For each sample, we estimated the expression of full-length STMN2 using all RNA-Seq reads covering known STMN2 exons, whereas the truncated STMN2 isoform was quantified using high-confidence spliced RNA-Seq reads that unambiguously connected STMN2 exon 1 with the cryptic exon 2a (exon 1-exon 2a) (Figure 3A). We found that full-length STMN2 was highly expressed in both cortical and cerebellar tissues (median transcripts per million [TPM] in frontal cortex $=58.0$ and in cerebellum $=73.1)$ compared with expression levels in spinal cord (median TPM in lumbar spinal cord $=6.7$ ), presumably because its expression is restricted to neurons and because of the higher white matter composition in the spinal cord (Supplemental Figure 4).

We then estimated the expression of truncated STMN2 in the NYGC ALS cohort in disease groups across tissues. At least 2 reads spanning the exon 1-exon 2a junction could be detected in 398 of the 1659 samples in the data set (24.0\%) (Supplemental Figure 5). Samples that expressed the splice junction did so at a median read count of 6 ( 0.13 reads per million) and a maximum read count of 120 (2.5 reads per million) (Supplemental Figure 6). We therefore chose to model truncated STMN2 expression as a binary outcome, with a detection threshold of 2 junction reads uniquely mapped across the intron boundary.

Truncated STMN2 was present in disease subtypes known to have TDP-43 pathology, as it was detected in the frontal cortex of $82.4 \%$ of patients with FTLD-TDP but was absent in those with FTLD-FUS or FTLD-tau and in controls (Figure 3B). In ALS lumbar spinal cord, we detected truncated STMN2 in $62.2 \%$ of patients with ALS with presumed TDP-43 pathology (ALS-TDP) but not in patients with ALS-SOD1 or in control individuals (Figure 3C).

We observed an exquisite specificity of truncated STMN2 expression in tissues known to have TDP-43 pathology. In patients with FTLD-TDP, for whom frontal cortex, temporal cortex, and cerebellum data sets were available, we detected truncated STMN2 in frontal and temporal cortices but not in cerebellum (Figure 3D). In ALS-TDP tissues, which also included hippocampus, temporal cortex, motor cortex, occipital cortex, and spinal cord samples, truncated STMN2 expression was most prevalent in the spinal cord, followed by the motor cortex and frontal cortex, with little to no expression detected in the occipital cortex or cerebellum, 
A

$$
\text { qRT-PCR }
$$

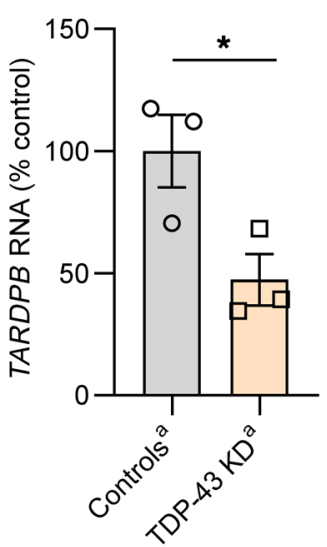

D

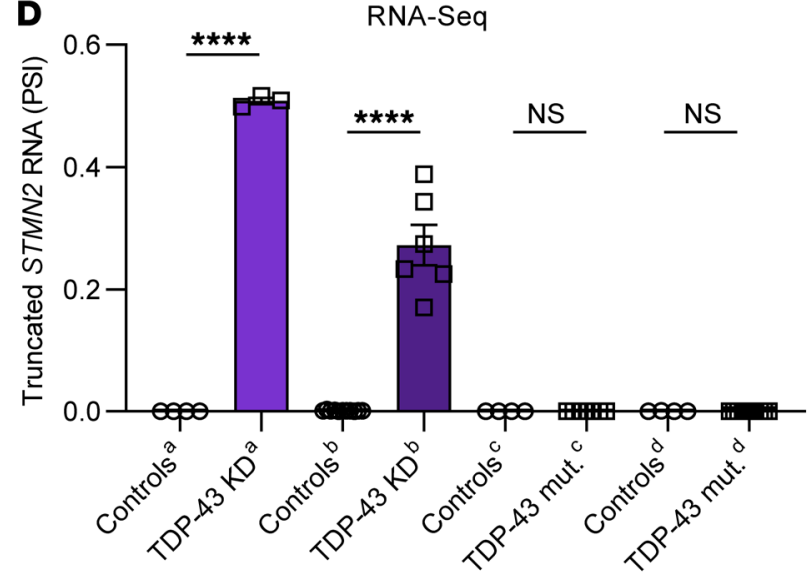

B

qRT-PCR
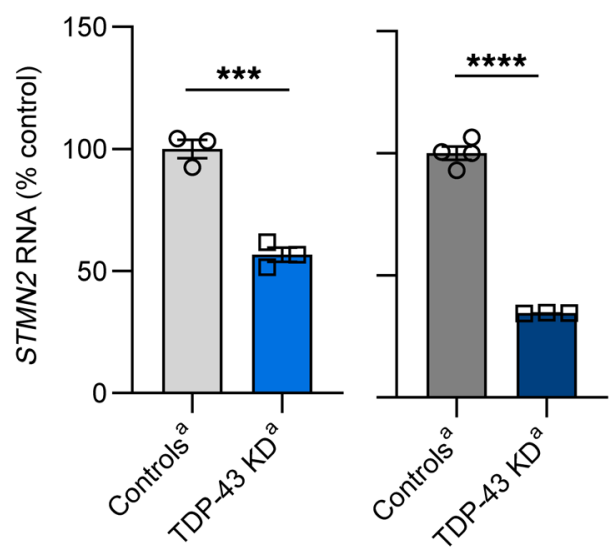

$\mathbf{E}$
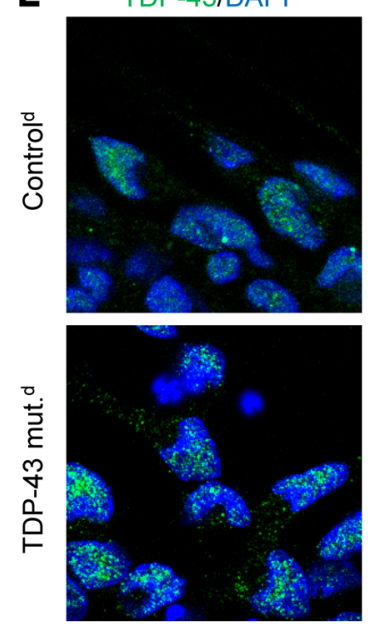

C $\quad$ qRT-PCR

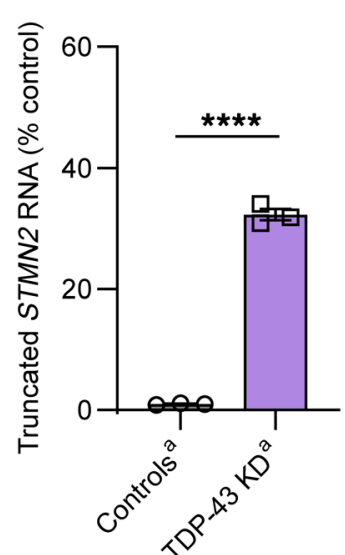

ChAT/DAPI
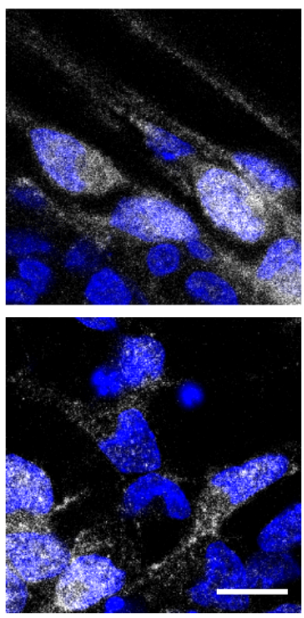

Figure 2. Truncated STMN2 RNA is elevated in iPSC-derived neurons with reduced levels of TDP-43. (A-C) iPSC-derived cortical neurons constitutively expressing CRISPR inactivation machinery (data set labeled "a") were transduced with a lentivirus expressing a sgRNA against TARDBP (TDP-43 KD) or a control sgRNA (Controls) and subjected to analysis by qRT-PCR ( $n=3$ TDP-43 KD, $n=3$ controls) and/or RNA-Seq ( $n=3$ TDP-43 KD, $n=4$ controls). The graphs show a decrease in TARDBP transcripts (A), a reduction of the full-length STMN2 transcript (B), and an increase in the truncated STMN2 transcript (C) upon TDP-43 depletion. (D) Detection of STMN2 cryptic exon 2a inclusion expressed as PSI by RNA-Seq in various iPSC-derived neurons: iPSC-derived cortical neurons (data set labeled "a") described above and performed in this study; iPSC-derived motor neurons treated with TARDBP siRNA (TDP-43 KD) or control siRNA (controls) from Klim et al. (data set labeled "b") ( $n=6$ TDP-43 KD, $n=11$ controls) (7); iPSC-derived motor neurons from patients with TARDBP mutations (TDP-43 mut.) or controls from 2 independent groups in Edinburgh (data set labeled " $c$ ") ( $n=7$ TDP-43 KD, $n=4$ controls) and Oxford (data set labeled "d") ( $n=10$ TDP-43 KD, $n=4$ controls) (72). Data are presented as the mean \pm SEM and were normalized to the control groups in $\mathbf{A}$ and $\mathbf{B}$ (set to $100 \%$ ). ${ }^{*} P<0.05$, ${ }^{* * *} P<0.005$, and ${ }^{* * * *} P<0.001$, by Student's $t$ test (A-C) or 1-way ANOVA (D). (E) Representative images of iPSC-derived motor neurons from patients with TARDBP mutations and control subjects from a group in Oxford (data set labeled "d" in Figure 1D) showed similar nuclear-cytoplasmic distribution of TDP-43 (green) on day 30 of differentiation (DAPI in blue, choline acetyltransferase [ChAT] in white). Scale bar: $10 \mu \mathrm{m}$. All panels of Figure 2E are reshown in Supplemental Figure 3A. Additional data associated with this figure can also be found in Supplemental Figure 3. See also the complete unedited blots in the supplemental material.

respectively (Figure 3E and Supplemental Figure 5). As anticipated, patients with concurrent FTD and ALS (ALS-FTLD) had truncated STMN2 expression in frontal and temporal cortices, as well as in the motor cortex and spinal cord (Supplemental Figure 7A). In contrast, patients with ALS-AD strongly resembled patients with ALS-TDP in their truncated STMN2 detection patterns, with most truncated STMN2 expression found in spinal cord and reduced or no expression in cortical brain regions (Supplemental Figure 7B). Together, these findings demonstrate that truncated STMN2 RNA is detected in diseases and tissues characterized by TDP-43 pathology, supporting its potential as a marker for TDP-43 dysfunction.
Truncated STMN2 RNA expression is elevated in FTLD-TDP frontal cortex. Given that we detected truncated STMN2 RNA in patients with FTLD-TDP but not in those with FTLD-FUS or FTLD-tau (Figure $3 \mathrm{~B}$ ), this aberrant splicing event may provide a means to identify patients with FTD with marked TDP-43 pathology. To further examine the relationship between TDP-43 and STMN2 in patients with FTD, we used the NanoString PlexSet platform to systematically quantify truncated and full-length STMN2 RNA levels in frontal cortex tissue from a cohort of 238 patients with FTD with immunohistologically confirmed TDP-43-immunoreactive inclusions, a cohort of 33 cognitively normal controls who were TDP-43 negative 
A

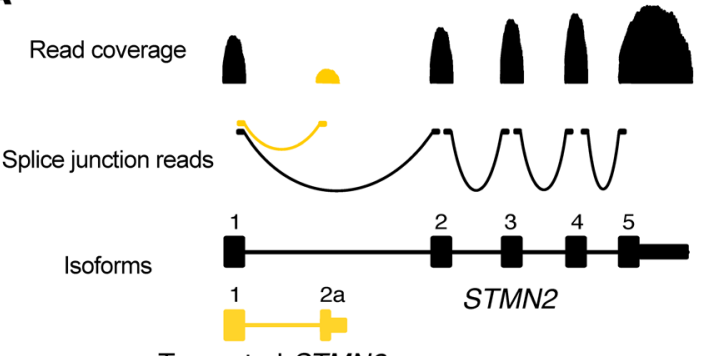

Truncated STMN2

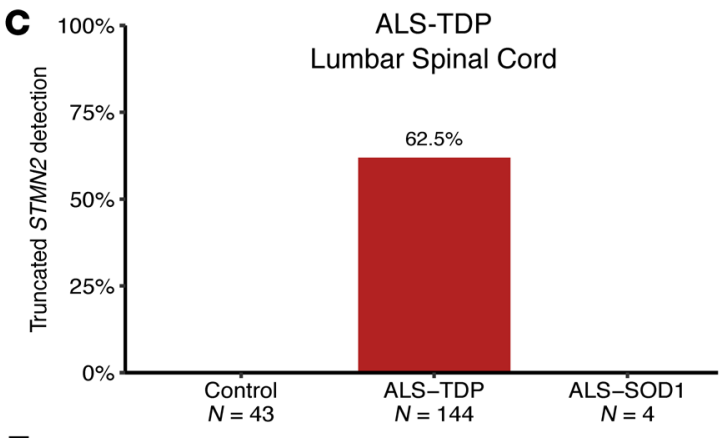

B

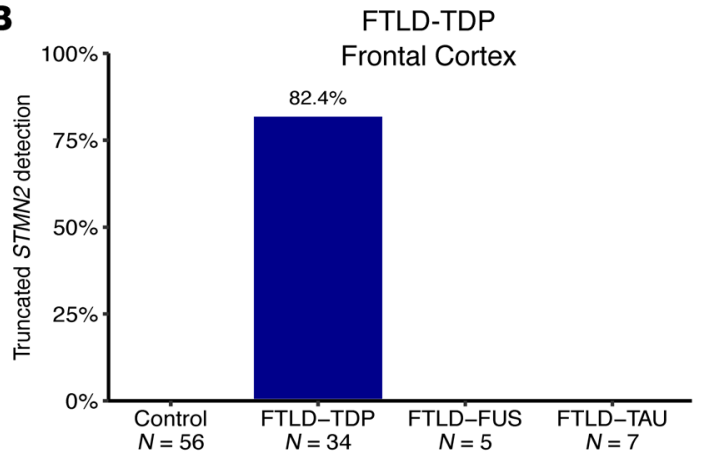

D

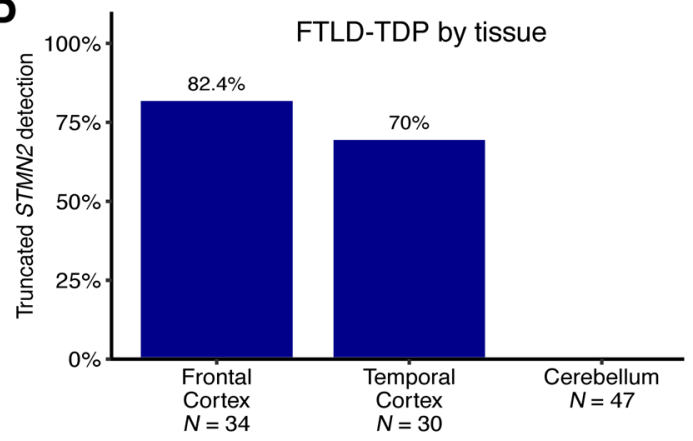

$\mathbf{E}$

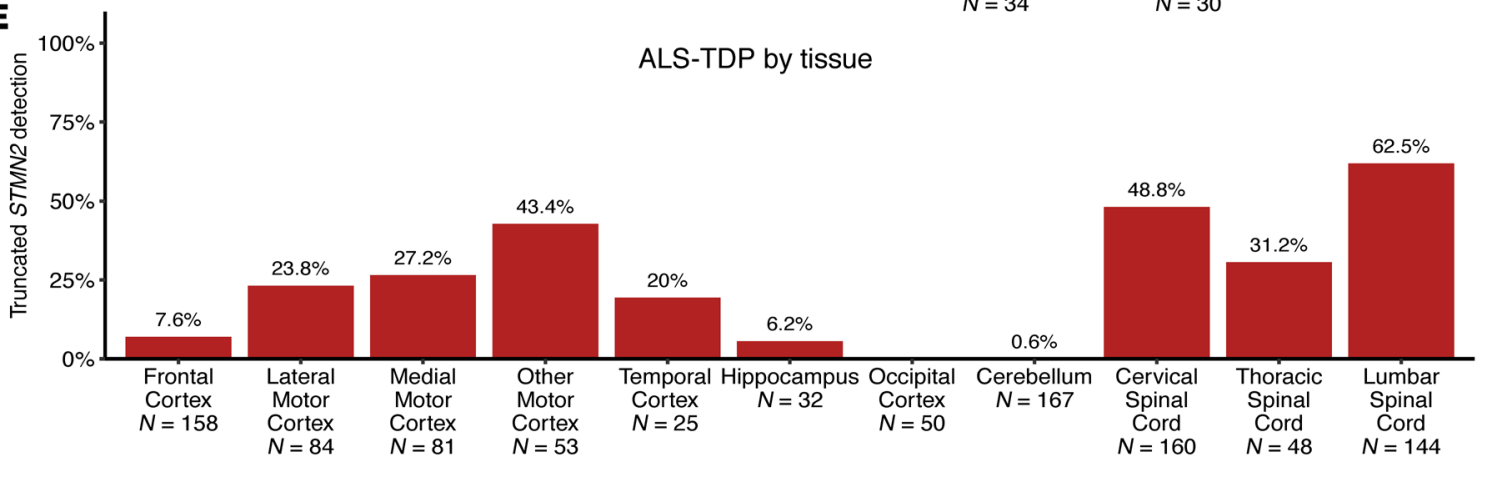

Figure 3. Truncated STMN2 RNA is detected in bulk RNA-Seq from FTLD/ALS tissues with TDP-43 pathology. (A) The NYCC ALS Consortium RNA-Seq data set was analyzed for the presence of truncated STMN2 transcripts, which were identified by RNA-Seq reads spanning the exon 1-exon 2a splice junction. (B-E) Bar graphs represent the proportion of individuals (percentage) with at least 2 reads spanning the exon 1-exon 2a junction. The number of individuals for the samples of the indicated tissues/diseases is indicated. (B) Truncated STMN2 was detected in the frontal cortex of patients with FTLDTDP but not in control subjects or patients with FTLD-FUS or FTLD-tau. (C) In the lumbar spinal cord of patients with ALS, truncated STMN2 RNA was only detected in ALS-TDP samples but not in ALS-SOD1 or control samples. (D) Among FTLD-TDP tissues, truncated STMN2 was only detected in those known to have TDP-43 pathology (frontal and temporal cortex), whereas (E) within ALS-TDP tissues, truncated STMN2 RNA was seen in motor cortex and spinal cord tissues but not in regions without TDP-43 pathology.

(henceforth referred to as controls), and a cohort of 41 patients with PSP (Table 1). The latter cohort of patients were characterized by tau pathology and neurodegeneration in the frontal cortex.

As mentioned, FTLD-TDP can be further classified on the basis of morphology and distribution of TDP-43 inclusions. As such, our cohort of patients with FTLD-TDP were classified into different TDP-43 pathological subtypes: 117 patients were type A (27 and 34 patients carried a mutation in C9orf72 or GRN, respectively), 66 were type B (21 and 1 carried a mutation in C9orf 72 or $G R N$, respectively), 43 were type C ( 2 and 1 carried a mutation in C9orf72 or GRN, respectively), and 2 were type D (both patients had VCP mutants) (Table 1). In addition to conducting our analyses of all FTLD-TDP cases combined, we also evaluated whether truncated STMN2 accumulation was preferentially associated with a particular TDP-43 subtype. In the frontal cortex, we found that truncated STMN2 RNA levels were significantly elevated in all patients with FTLD-TDP compared with levels in the control subjects without disease or those with PSP disease in both unadjusted analysis $(P<0.001)$ (Figure $4 \mathrm{~A}$, Table 2 , and Supplemental Table 2) and analysis adjusted for age at death, sex, and RNA integrity number (RIN) $(P=0.009$ vs. controls, $P<0.001$ vs. patients with PSP) (Figure 4A, Table 2, and Supplemental Table 2). We also noted that the levels of truncated STMN2 in individuals with PSP were significantly lower than those in control subjects $(P=0.028$, adjusted analyses for age, sex, and RIN), possibly because of the neuronal loss in the patients with PSP.

As in all patients with FTLD-TDP combined, truncated STMN2 RNA levels in the frontal cortex were increased in FTLD-TDP for each TDP-43 type (A-D) compared with levels in patients with PSP in both unadjusted and adjusted analyses (Table 2 and Sup- 
Table 1. Patient characteristics in the postmortem cohort

\begin{tabular}{|c|c|c|c|c|c|c|c|}
\hline Variable & $\begin{array}{l}\text { Controls } \\
(n=33)\end{array}$ & $\begin{array}{c}\text { PSP } \\
(n=41)\end{array}$ & $\begin{array}{l}\text { All FTLD-TDP } \\
(n=238)\end{array}$ & $\begin{array}{l}\text { FTLD-TDP type A } \\
\quad(n=117)\end{array}$ & $\begin{array}{c}\text { FTLD-TDP type B } \\
(n=66)\end{array}$ & $\begin{array}{l}\text { FTLD-TDP type C } \\
\quad(n=43)\end{array}$ & $\begin{array}{l}\text { FTLD-TDP type D } \\
(n=2)\end{array}$ \\
\hline Age at death (yr) & $82(55,99)$ & $69(52,86)$ & $72(49,100)$ & $76(50,100)$ & $67(49,98)$ & $74(60,84)$ & $63(61,64)$ \\
\hline \multicolumn{8}{|l|}{ Sex } \\
\hline Female & $13(39.4 \%)$ & $20(48.8 \%)$ & $108(45.4 \%)$ & $54(46.2 \%)$ & $29(43.9 \%)$ & $20(46.5 \%)$ & $1(50.0 \%)$ \\
\hline Survival after onset (yr) & NA & $7.0(4.0,14.0)$ & $7.0(0.6,25.0)$ & $7.0(1.0,25.0)$ & $4.0(0.6,20.0)$ & $10.6(4.0,25.0)$ & $8.5(7.0,10.0)$ \\
\hline Age at onset (yr) & NA & $61.5(46.0,79.5)$ & $64.5(40.0,85.8)$ & $67.8(40.0,85.8)$ & $61.4(44.0,85.7)$ & $62.7(46.0,76.0)$ & $54.1(54.0,54.2)$ \\
\hline
\end{tabular}

The sample median (minimum, maximum) is given for continuous variables. Information was unavailable regarding TDP-43 subtype (all FTLD-TDP: $n=10$ ), age at death (all FTLD-TDP: $n=1$ ), p-TDP-43 burden in the frontal cortex (all FTLD-TDP: $n=1$ ), survival after onset (PSP: $n=4$; all FTLD-TDP: $n=20$ ), and age at onset (PSP: $n=4$; all FTLD-TDP: $n=20$ ). Among the patients with FTLD-TDP, 50 had C9orf72 mutations ( $n=27$ TDP-43 type A, $n=21$ TDP-43 type B, $n=2$ TDP-43 type C), 36 had GRN mutations ( $n=34$ TDP-43 type A, $n=1$ TDP-43 type B, $n=1$ TDP-43 type C), and 2 had VCP mutations (TDP-43 type D).

plemental Table 2). Compared with controls, truncated STMN2 levels were significantly increased in patients with FTLD-TDP types A, B, and C, but not in those with type D, in unadjusted analyses (Table 2 and Supplemental Table 2), although the levels only in those with type A FTLD-TDP remained significant in analyses adjusted for age, sex, and RIN (Table 2 and Supplemental Table 2).

We observed that truncated STMN2 levels in the frontal cortex discriminated between the patients with FTLD-TDP and the PSP control subjects, with an area under the receiver operating characteristic curve (AUC) of 0.76 (95\% CI=0.69-0.84) (Table 2). Frontal cortex truncated STMN2 also discriminated between FTLD-TDP patients and cognitively normal controls with a more modest AUC of 0.67 (95\% CI=0.57-0.78) (Supplemental Table 2). Furthermore, truncated STMN2 levels similarly discriminated between controls (cognitively normal or PSP disease controls) and patients with FTLD-TDP of all TDP-43 types, most prominently for types A, C, and D (Table 2 and Supplemental Table 2). In contrast, truncated STMN2 levels did not effectively distinguish patients with PSP from control subjects (AUC: 0.60, 95\% CI=0.47-0.73).

The increase in truncated STMN2 levels in FTLD-TDP frontal cortex was paired with a decrease in full-length STMN2 levels $(P<0.001)$ (Figure 4B and Supplemental Table 3). Of interest, fulllength STMN2 expression was also decreased in patients with PSP compared with expression levels in controls $(P<0.001)$ (Figure $4 \mathrm{~B}$ and Supplemental Table 3), probably because of neurodegeneration in this region. Full-length STMN2 levels also distinguished control subjects from patients with FTLD-TDP (AUC: 0.74) and PSP (AUC: 0.83), but were not as effective in distinguishing patients with FTLD-TDP from those with PSP (AUC: 0.60, 95\% CI=0.51-0.68; Supplemental Table 3).

Taken together, our data show that truncated STMN2 RNA levels were significantly elevated in patients with FTLD-TDP compared with levels in controls and may be used to distinguish FTD patients with TDP-43 pathology from both cognitively normal individuals and disease controls.

Truncated STMN2 RNA associates with p-TDP-43 burden in the frontal cortex of patients with FTLD-TDP. That truncated STMN2 levels were elevated in the frontal cortex of patients with FTLDTDP but not in those with PSP (Figure 4A) or other forms of FTLD
(FTLD-tau, FTLD-FUS) or ALS (ALS-SOD1) (Figure 3) suggests that truncated STMN2 production is caused by a loss of TDP-43 nuclear function resulting from its mislocalization to the cytoplasm and/or its sequestration into inclusions - hallmark features of FTLD-TDP. To evaluate this possibility further, we examined whether full-length or truncated STMN2 RNA levels in the frontal cortex correlated with p-TDP-43 accumulation. To this end, we prepared urea-soluble protein lysates from the same tissues used to measure STMN2 isoforms and quantified p-TDP-43 in these lysates using a previously described immunoassay (32-34). Although the levels of full-length STMN2 did not associate with p-TDP-43 levels ( $\beta$ : 0.02, $P=0.53$ ) (Supplemental Table 4), higher $\mathrm{p}-\mathrm{TDP}-43$ levels were associated with higher truncated STMN2 RN A levels (Figure 4C) in both unadjusted analyses ( $\beta$ : 0.20 , $P<0.001$ ) (Table 3 ) and in a multivariable analysis adjusted for age, sex, and TDP-43 subtype ( $\beta$ : 0.19, $P<0.001$ ) (Table 3$)$. Taken together, our data suggest that truncated STMN2 RNA, but not full-length STMN2, accumulates in brain regions marked by TDP43 pathology and associates with $\mathrm{p}$-TDP-43 burden.

Truncated STMN2 RNA levels associate with an earlier age of FTLD-TDP onset. We additionally examined whether truncated or full-length STMN2 levels in the frontal cortex associate with clinical characteristics of individuals with FTLD-TDP. We found a significant inverse correlation between truncated STMN2 RNA levels and age at disease onset (Figure 4D) in analyses, adjusting for sex, age at death, and TDP-43 type and after correcting for multiple testing $(P=0.005)$ (Table 3). However, survival after onset did not associate with truncated STMN2 RNA levels, after correcting for age at onset, sex, and TDP-43 subtype ( $P=0.32$ ) (Table 3$)$. Of interest, we observed a nominal but not statistically significant association with lower truncated STMN2 levels in males ( $\beta$ : $-0.35, P=0.034$ in adjusted analyses for age and TDP-43 type) (Supplemental Table 4). In contrast, full-length STMN2 levels failed to associate with clinical features of FTLD-TDP, including age of onset and survival (Supplemental Table 4).

\section{Discussion}

FTLD, the neuropathological substrate of FTD, is the second most common cause of early-onset dementia (35). Although no effective treatment is available, several therapeutic approaches 
A
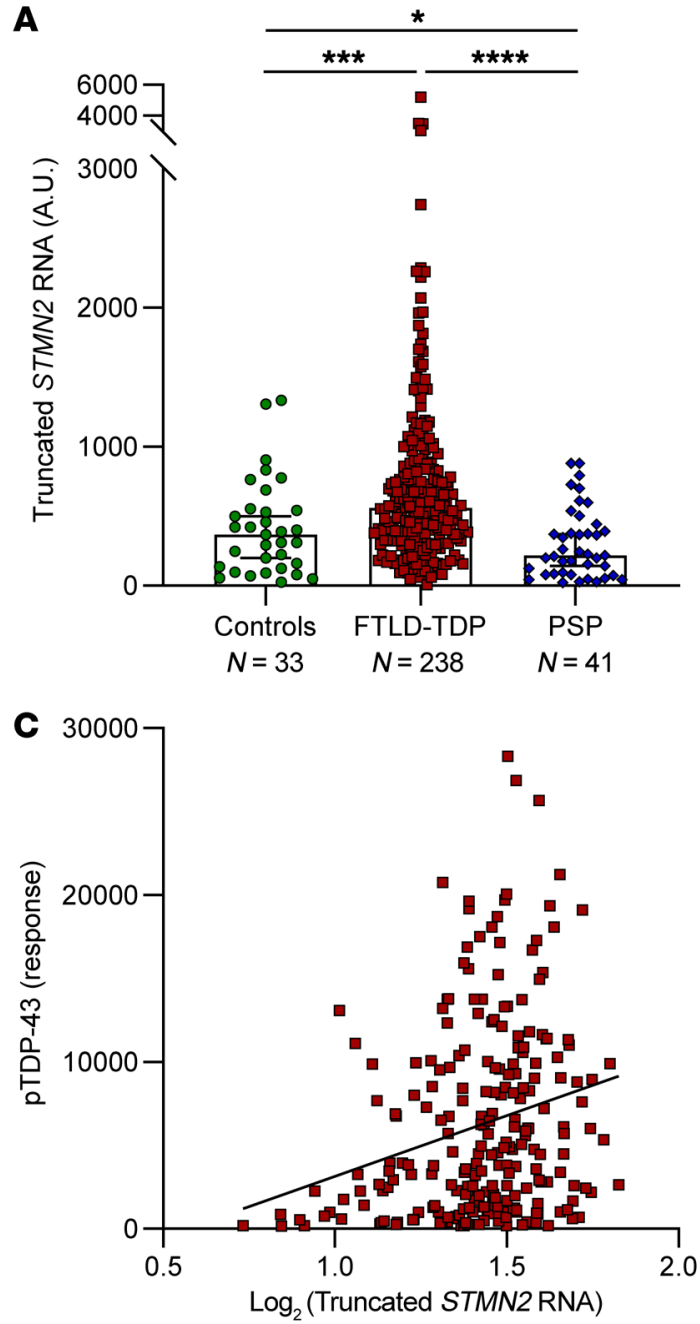

B
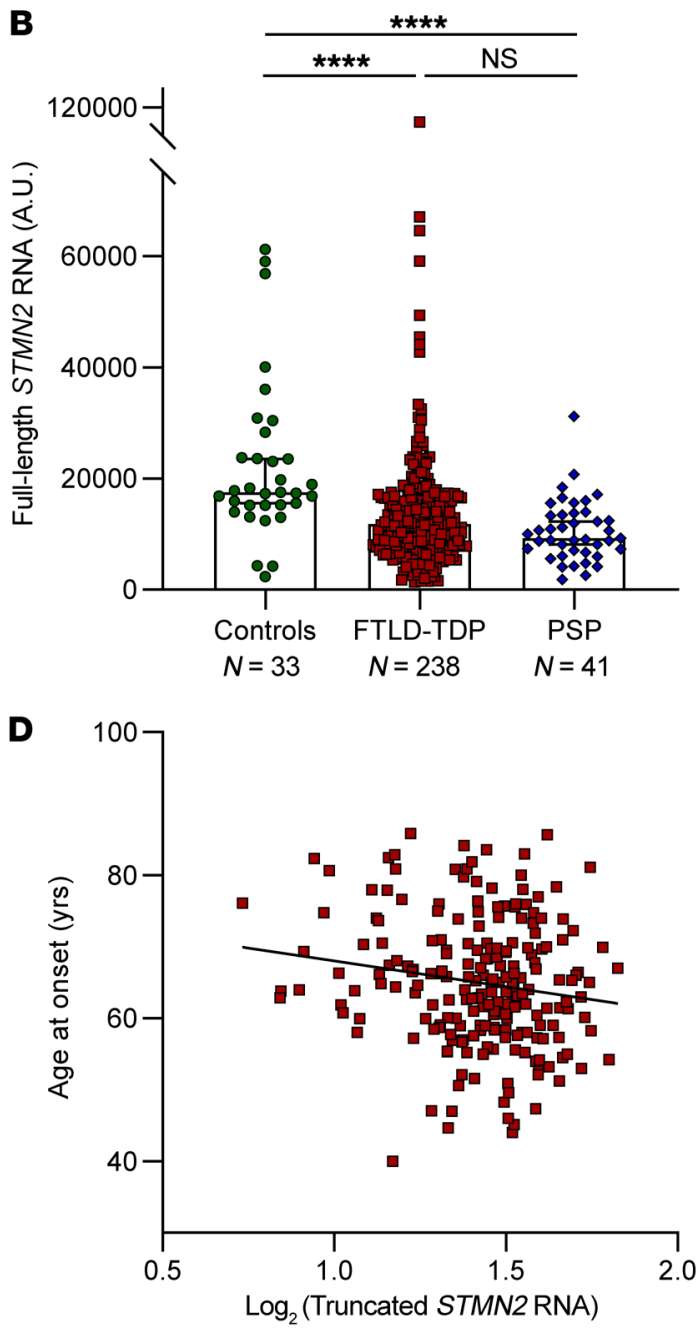

Figure 4. Truncated STMN2 is elevated in FTLD-TDP and associates with higher P-TDP-43 burden and earlier age of FTD onset. (A and B) Truncated and fulllength STMN2 levels were measured in RNA extracted from frontal cortex of control subjects and FTLD-TDP and PSP patients, using the NanoString PlexSet platform. (A) A significant accumulation of truncated STMN2 RNA was detected in patients with FTLD-TDP but not in controls (see also Supplemental Table 2) or patients with PSP (see also Table 2). (B) Full-length STMN2 levels were significantly decreased in FTLD-TDP and PSP patients (see also Supplemental Table 3). Data are presented as the median with a $95 \% \mathrm{Cl}$, and $P$ values were determined using linear regression models that were adjusted for age at death, sex, and RIN. ${ }^{*} P<0.05,{ }^{* *} P<0.005,{ }^{* * *} P<0.001$. (C and $\left.\mathbf{D}\right)$ In our FTLD-TDP cohort $(n=238)$, significant correlations were observed between truncated STMN2 RNA levels and (C) a higher burden of p-TDP-43 and (D) an earlier age of disease onset. See Table 3 for the correlation coefficients.

are being investigated to target TDP- 43 and tau, the 2 proteins most commonly associated with the major pathological FTLD subtypes. Their testing in clinical trials, however, faces a considerable barrier: our inability to determine the underlying protein pathology in living patients with FTD. Although the pathomechanisms driven by TDP- 43 and tau differ, the clinical symptoms of FTD patients with TDP- 43 or tau pathology overlap. Ultimately, this hinders the selection of patients with sporadic FTD who are suitable for a particular clinical trial. Since there is a clear need for biomarkers to identify FTD patients with TDP-43 pathology, we evaluated whether truncated STMN2 could serve as a proxy for TDP-43 pathology. Using RNA-Seq and RNA quantification techniques, we show that truncated STMN2 RNA levels were elevated in human iPSC-derived neurons depleted of TDP-43 and in bulk tissue from FTD and ALS patients with TDP-43 pathology. We did not detect truncated STMN2 in iPSC-derived neurons with pathogenic TARDBP mutations where no change in TDP-43 levels, localization, or aggregates was detected, supporting the fact that loss of TDP-43 nuclear function, and not splicing changes induced by pathogenic mutations, were driving this event. We were unable to assess postmortem tissue from patients carrying TARDBP mutations, which are rare in the FTD and ALS populations, as a group, but given that these patients develop florid TDP-43 pathology, we expected truncated STMN2 to be present at levels similar to those in patients with sporadic ALS or FTLD-TDP. Additionally, we found that truncated STMN2 RNA accumulated in the frontal cortex of patients with FTLD-TDP but not in control subjects or patients with PSP and was significantly correlated with p-TDP-43 levels and an earlier age of disease onset. Taken together, our data support the notion that truncated STMN2 production results from TDP-43 loss of function. 


\section{Table 2. Comparisons of truncated STMN2 RNA in frontal cortex between patients with FTLD-TDP and patients with PSP.}

Regression coefficients, $95 \% \mathrm{Cls}$, and $P$ values were determined using linear regression models, where the levels of truncated STMN2 RNA in the frontal cortex were considered on the base 2 logarithmic scale. Regression coefficients were interpreted as the difference in mean levels of truncated STMN2 RNA in the frontal cortex (on the base 2 logarithmic scale) between the given group of patients with disease and patients with PSP. $P$ values of 0.010 or less were considered statistically significant after applying a Bonferroni's correction for multiple testing.

As mentioned above, 2 independent reports recently revealed that TDP-43 regulates STMN2 expression and that TDP-43 depletion produces a truncated, nonfunctional STMN2 variant $(7,8)$. Increased truncated STMN2 RNA levels were detected in spinal cord tissue of 17 patients with ALS but not in that of 6 controls, and ISH studies found truncated STMN2 RNA in lower and upper motor neurons in 5 patients with ALS (8). We thus evaluated truncated STMN2 as a proxy TDP-43 activity marker for FTD using postmortem RNA-Seq data across 11 tissues available from a large cohort of FTD, ALS, and FTLD-ALS patients. Notably, our RNA-Seq analyses detected the STMN2 cryptic exon that gives rise to truncated STMN2 only in FTD and ALS patients with TDP-43 pathology. In addition, the presence of abnormal STMN2 was highly specific to brain regions characterized by TDP- 43 pathology.

In addition to our analyses of RNA-Seq data, we directly measured truncated STMN2 RNA in postmortem frontal cortex tissue from patients with FTLD-TDP, which again revealed an aberrant accumulation of truncated STMN2 in comparison with controls and individuals with PSP. In fact, we found that truncated STMN2 RNA levels distinguished between patients with FTLDTDP and those with PSP, with an area under the ROC curve of 0.76. As anticipated, truncated STMN2 accumulation in patients with FTLD-TDP was accompanied by a decrease in full-length STMN2, as was observed in cell culture models $(7,8)$. Full-length STMN2 was also decreased in patients with PSP, which were confirmed to lack TDP-43 pathology. Given that STMN2 is expressed by neurons and its expression is abundant in the frontal cortex, the decrease in full-length STMN2 in patients with PSP likely reflects neurodegenerative neuronal loss. Indeed, a recent report found that full-length STMN2 RNA levels were also decreased in the brains of patients with Parkinson's disease (36), possibly arguing for the use of STMN2 levels as a neurodegenerative disease biomarker. It is also possible that, aside from TDP-43, various other factors regulate full-length STMN2 expression. This could explain why full-length STMN2 levels did not correlate with p-TDP-43 burden or with clinical features.

The accumulation of truncated STMN2 RNA in the frontal cortex of patients with FTLD-TDP but not of patients with PSP indicates that truncated STMN2 production is caused by patho- logical changes in TDP-43. In line with this, we observed a significant association of truncated STMN2 RNA with p-TDP-43 protein levels $(P<0.001$, Table 3$)$, which remained statistically significant after controlling for multiple variables (age, sex, and TDP-43 subtype; $P<0.001$, Table 3 ). However, it is possible that p-TDP-43 levels alone may not be a perfect corollary for nuclear TDP-43 depletion. Of interest, the levels of truncated STMN2 RNA did not differ among different TDP-43 subtypes, suggesting that truncated STMN2 levels are elevated in all types of TDP-43 pathology.

Importantly, higher truncated STMN2 RNA levels in the frontal cortex at death were significantly associated with an earlier age at disease onset $(P=0.005$, Table 3$)$, but we found no association with survival after disease onset. Given that truncated STMN2 RNA serves as a proxy for TDP-43 dysfunction, this earlier age of onset in patients with higher postmortem truncated STMN2 RNA levels could point to more severe defects in RNA metabolism resulting from aberrant TDP-43 function, including loss of STMN2 function.

Strengths of our study include the use of samples from a clinically well-characterized, immunohistologically confirmed cohort of 238 patients with FTLD-TDP from which high-integrity RNA was extracted from the frontal cortex, and the use of quantitative measurements of truncated STMN2 RNA and p-TDP-43 protein. Moreover, we evaluated multiple neuroanatomical regions in bulk tissue RNA-Seq from a large data set that included 1659 FTD and ALS patients with and without TDP-43 pathology. Further, our study also used 3 different approaches (RNA-Seq, NanoString, and qRT-PCR, the latter of which was also performed to validate truncated STMN2 in patients with FTLD-TDP, as shown in Supplemental Figure 9) that have different sensitivity and specificity linked to the methodology but that all detect significant differences in truncated STMN2, highlighting the reproducibility of the finding. Our study also has some limitations. While we specifically designed our study to evaluate truncated STMN2 as a marker of TDP-43 pathology - information important to establish its value as a candidate biomarker - we did not measure the translation product from this truncated variant because of a lack of commercially available antibodies. At present, it is unclear whether 
Table 3. Associations of truncated STMN2 with p-TDP-43 and clinical characteristics in FTLD-TDP frontal cortex

Association with frontal cortex truncated STMN2 RNA

\begin{tabular}{|c|c|c|c|c|c|}
\hline & \multicolumn{2}{|c|}{ Unadjusted analysis } & \multicolumn{2}{|c|}{ Multivariable analysis } & \multirow[b]{2}{*}{ Multivariable model adjustments } \\
\hline Variable & $\begin{array}{l}\text { Regression coefficient } \\
(95 \% \mathrm{Cl})\end{array}$ & $P$ value & $\begin{array}{l}\text { Regression coefficient } \\
(95 \% \mathrm{Cl})\end{array}$ & $P$ value & \\
\hline p-TDP-43 (doubling) & $0.20(0.12,0.28)$ & $<0.001$ & $0.19(0.09,0.28)$ & $<0.001$ & Age at death, sex, TDP-43 subtype \\
\hline FTLD-TDP type A & 0.00 (reference) & NA & 0.00 (reference) & NA & \\
\hline FTLD-TDP type B & $-0.19(-0.57,0.19)$ & 0.33 & $-0.31(-0.72,0.10)$ & 0.14 & \\
\hline Age at onset (10-yr increase) & $-0.23(-0.41,-0.04)$ & 0.015 & $-0.29(-0.49,-0.09)$ & 0.005 & Sex and TDP-43 subtype \\
\hline Survival after onset (5-yr increase) & $0.12(-0.06,0.30)$ & 0.19 & $0.10(-0.09,0.29)$ & 0.32 & Age at onset, sex, and TDP-43 subtype \\
\hline \multicolumn{6}{|l|}{ Sex } \\
\hline Male & $-0.37(-0.68,-0.06)$ & 0.019 & $-0.35(-0.67,-0.03)$ & 0.034 & Age at death and TDP-43 subtype \\
\hline Female & 0.00 (reference) & NA & 0.00 (reference) & NA & \\
\hline
\end{tabular}

Regression coefficients, 95\% Cls, and $P$ values were determined using linear regression models, where frontal cortex truncated STMN2 RNA was considered on the base 2 logarithmic scale. Regression coefficients were interpreted as the change in mean frontal cortex truncated STMN2 RNA (on the base 2 logarithmic scale) corresponding to the presence of the indicated characteristic (categorical variables) or the increase shown in parentheses (continuous variables). $P$ values of 0.010 or less were considered statistically significant after applying a Bonferroni's correction for multiple testing.

the predicted peptide of only 16 amino acids is translated from truncated STMN2 RNA. Nevertheless, our findings indicate that the development of novel tools (antibodies, immunoassays) for the detection of truncated STMN2 protein and additional studies to evaluate the role of truncated STMN2 RNA and protein in disease pathogenesis and as biomarkers are well merited. Critical next steps to hasten the translation of this finding to the clinic include determining whether truncated STMN2 RNA or protein is detected in the biofluids of patients with FTD (e.g., spinal fluid and blood) and, if so, whether they discriminate between FTD subtypes (i.e., with or without TDP-43). Although expression of truncated STMN2 in affected postmortem tissues can distinguish FTLD-TDP from PSP cases, which has important implications, as it supports truncated STMN2 as a viable proxy for the presence of TDP-43 pathology, the ability to discriminate between FTLD-TDP and PSP patients was only modest (AUC: 0.76). RNA degradation invariably occurs in postmortem brain tissues, possibly contributing to this modest AUC and potentially pointing toward the possibility of detecting truncated STMN2 in CSF or blood, which are typically frozen immediately after collection, to discriminate between patient groups. Nonetheless, sensitive methods will be needed to detect truncated STMN2 RNA and/or protein in human biofluids. Additionally, determining at what point during the course of disease truncated STMN2 levels begin to be upregulated will have to be evaluated and will probably require longitudinal collections of CSF and blood first from patients with ALS, who are almost certain to develop TDP-43 pathology, and then from individuals with FTD or FTD/ALS harboring genetic variants associated with TDP-43 dysfunction.

If, as anticipated, truncated STMN2 RNA or protein is detected in the biofluids of FTD patients with TDP-43 pathology, it may have the potential to similarly identify those patients with AD who have TDP- 43 pathology. Indeed, up to $67 \%$ of patients with
AD develop TDP-43 inclusions, and, compared with $\mathrm{AD}$ patients without TDP-43 pathology, they are cognitively and functionally worse, suggesting that abnormal TDP- 43 causes a modified AD phenotype (37-46). Our work also highlights how future means to detect truncated STMN2 RNA or protein in biofluids may provide important information on disease activity and therefore be useful in assessing drug efficacy in clinical trials. Thus, these endeavors are expected to increase the likelihood of success in developing effective therapeutics and improving the care of patients with TDP-43 proteinopathies.

\section{Methods}

RNA extraction, complementary DNA synthesis, and qRT-PCR in iPSC-derived neurons. iPSC-derived neurons were harvested, and cell pellets were lysed in TRI reagent (Zymo Research). Then, total RNA was extracted following the manufacturer's instructions using the Direct-zol RNA Miniprep Kit (Zymo Research), and 850 ng RNA was used for RT-PCR. RT-PCR of RNA samples was performed using the High Capacity cDNA Transcription Kit with random primers (Applied Biosystems), and qRT-PCR was performed using SYBR GreenER qPCR SuperMix (Invitrogen, Thermo Fisher Scientific). Samples were run in triplicate, and qRT-PCRs were run on a Prism 7900HT Fast Real-Time system (Applied Biosystems). Relative quantification was determined using the $\triangle \Delta \mathrm{Ct}$ method and normalized to the endogenous controls RPLPO and GAPDH. The following primers and their sequences were used: STMN2 forward: 5'-AGCTGTCCATGCTGTCACTG-3', STMN2 reverse: 5'-GGTGGCTTCAAGATCAGCTC-3'; truncated STMN2 forward: 5'-GGACTCGGCAGAAGACCTTC-3', truncated STMN2 reverse: 5'-GCAGGCTGTCTGTCTCTCTC-3'; TARDBP forward: 5'-AATTCTGCATGCCCCAGA-3', TARDBP reverse: 5'-GAAGCATCTGTCTCATCCATTTT-3'; GAPDH forward: 5'-GTTCGACAGTCAGCCGCATC-3', GAPDH reverse: 5'-GGAATTTGCCATGGGTGGA-3'; and RPLPO forward: 5'-TCTACAACCCTGAAGTGCTTGAT-3', $R P L P O$ reverse: $5^{\prime}$-CAATCTGCAGACAGACACTGG-3'. 
RNA-Seq analyses from iPSC-derived neurons. Data from Klim et al. (7) were downloaded from the NCBI's Gene Expression Omnibus (GEO) database (GEO GSE121569). All iPSC cells lines were analyzed with the same analysis pipeline. Raw FASTQ files were trimmed using fastp (47) and aligned to the hy38 build (GRCh38.primary_assembly) of the human reference genome using STAR (2.7.2a) (48). Gene abundance for TARDBP and STMN2 was calculated with featureCounts (49) using gene models from GENCODE GTF, version 31, and converted to reads per kilobase per million (RPKM) using edgeR (50). The relative abundance of truncated STMN2 as a proportion of total STMN2 was expressed in terms of percent spliced in (PSI) (51). This was calculated by parsing the splice junction tables from STAR and taken as the percentage of all uniquely mapped STMN2 junction reads from exon 1 to exon 2 a over the sum of all junction reads coming from exon 1.

NYGC ALS Consortium cohort. Patients with FTD were classified according to a pathologist's diagnosis of FTD with TDP-43 inclusions (FTLD-TDP), tau inclusions (FTLD-tau), or FUS inclusions (FTLD-FUS). ALS samples were divided into the following subcategories using the available Consortium metadata: ALS with or without reported SOD1 mutations (ALS-TDP and ALS-SOD1); ALS with frontotemporal dementia (ALS-FTLD); and ALS with AD (ALS-AD). All non-SOD1 ALS samples were grouped as "ALSTDP" in this work for simplicity, although reporting of postmortem TDP-43 inclusions was not systematic and therefore not integrated into the metadata. Confirmed TDP-43 pathology postmortem was reported for all FTLD-TDP samples.

NYGC ALS Consortium RNA-Seq library preparation from bulk tissue RNA. The Consortium's sample processing has been, in part, previously described (52). In brief, RNA was extracted from flash-frozen postmortem tissue using TRIzol (Thermo Fisher Scientific) chloroform, followed by column purification (RNeasy Minikit, QIAGEN). RIN was assessed on a Bioanalyzer (Agilent Technologies). RNASeq libraries were prepared from 500 ng total RNA using the KAPA Stranded RNA-Seq Kit with RiboErase (KAPA Biosystems) for rRNA depletion and Illumina-compatible indexes (NEXTflex RNA-Seq Barcodes, NOVA-512915, PerkinElmer, and IDT for Illumina TruSeq UD Indexes, 20022370). Pooled libraries (average insert size: 375 bp) passing the quality criteria were sequenced either on an Illumina HiSeq 2500 (125 bp paired end) or an Illumina NovaSeq (100 bp paired end). Samples were subjected to extensive sequencing and RNA-Seq quality control metrics at the NYGC that are described below. Notably, a set of more than 250 markers was used to confirm tissue, neuroanatomical regions, and sex in the RNA-Seq data. Samples passing these metrics are available for distribution. The samples had a median sequencing depth of 42 million read pairs, with a range between 16 and 167 million read pairs.

Analyses of bulk tissue RNA-Seq data. Samples were uniformly processed using RAPiD-nf, an efficient RNA-Seq processing pipeline implemented in the NextFlow framework (53). Following adapter trimming with Trimmomatic (version 0.36) (54), all samples were aligned to the hg38 build (GRCh38.primary_assembly) of the human reference genome using STAR (2.7.2a) (48), with indexes created from GENCODE, version 30 (55). Gene expression was quantified using RSEM (1.3.1) (56). Quality control was performed using SAMtools (57) and Picard Tools (58), and the results were collated using MultiQC (59). Exploratory plots were created in R, version 3.6.0 (60), using dplyr, tidyr, stringr, ggplot2, patchwork, and ggpubr packages (61-63).
RNA-Seq samples were subjected to quality control modeled on the criteria of the Genotype Tissue Expression Consortium (64). Any sample failing 1 of the following sequencing metric thresholds was removed: a unique alignment rate of less than $90 \%$, ribosomal bases of greater than $10 \%$, a mismatch rate of greater than $1 \%$, a duplication rate of greater than $0.5 \%$, intergenic bases of less than $10.5 \%$, and ribosomal bases of greater than $0.1 \%$. Sex was verified using XIST and UTY expression. Tissue identity was confirmed using the expression of the cerebellar marker CBNL1 and the cortical marker NRGN. Spinal cord samples were confirmed by expression of the oligodendrocyte marker MBOP. Following quality control, 1905 samples of 1924 were retained for analysis. When comparing subgroups and tissues, only tissues with at least 50 samples and disease subgroups with at least 4 individuals were kept for analysis. This resulted in 1659 samples from 11 tissues and 439 donors.

Expression of the full-length STMN2 isoform was estimated using RSEM, summing the estimates of the 3 annotated isoforms in GENCODE, version 30, into a gene-level estimate of expression normalized in terms of TPM. Given the large size of the NYGC ALS cohort, RNA-Seq data were produced on 2 different sequencing platforms. Exploratory analyses showed a substantial batch effect in gene expression due to the 2 sequencing platforms. We therefore split the data set by sequencing platform, along with tissue and disease status. A comparison of ALS-TDP and non-neurological controls revealed that STMN2 had an inconsistent pattern of differential expression, with no tissue showing a marked difference in either platform (Supplemental Figure 4A). In addition, RNA degradation is known to affect the estimation of transcript abundance $(65,66)$, and we reasoned that systematic differences in RNA quality among samples sequenced on different platforms and between disease and control groups or tissues could confound our estimation of STMN2 expression. We compared the RIN values for each sample between the ALSTDP and control groups and found significant differences in RIN values in some comparisons (Supplemental Figure 4B). The small number of controls available limited further comparisons in some tissues. We then correlated the RIN with STMN2 expression and found a positive correlation in multiple tissues and on both sequencing platforms (Supplemental Figure 4C).

Quantifying STMN2 splicing from RNA-Seq data. For downstream analysis of STMN2 splice junction reads, a bioinformatics pipeline was created using Snakemake $(67,68)$. Uniquely mapped reads within the STMN2 locus were extracted from each sample using SAMtools. Any read marked as a PCR duplicate by Picard Tools was discarded. Splice junction reads were then extracted with RegTools (69) using a minimum of 8 bp as an anchor on each side of the junction and a maximum intron size of $500 \mathrm{~kb}$. Junctions from each sample were then clustered together using LeafCutter (70) with relaxed junction filtering (minimum total reads per junction $=30$, minimum fraction of total cluster reads $=$ 0.0001). This produced a matrix of junction counts across all samples. Using coordinates from the Klim (7) and Melamed (8) publications, the TDP-43-associated junction of the novel truncated STMN2 isoform was extracted from the matrix. A detection threshold of 2 or more uniquely mapped reads was applied. We found no association between truncated STMN2 detection and RIN, sequencing platform, or total library size (Supplemental Figure 8, A-C). The relationship between expression levels of full-length and truncated STMN2 was complex, with no overall detection of association across tissues (Supplemental Figure 8D). 
RNA extraction and NanoString analyses in postmortem tissues. Using the RNAeasy Plus Mini Kit (QIAGEN), RNA was extracted from frozen human postmortem frontal cortex tissues (33). RNA with a RIN of 7.0 or higher, as determined using an Agilent 2100 bioanalyzer (Agilent Technologies), was used to measure transcript levels using the NanoString PlexSet platform (250 ng human brain RNA, according to the manufacturer's instructions). Transcript level abundance was determined using nSolver 4.0 software (NanoString Technologies) and normalized to HTRP1, a housekeeping gene that was unaltered across disease subtypes in our data set and previously used to normalize human brain transcripts (71). The sequences of the NanoString probes used in this study are included in Supplemental Table 5.

p-TDP-43 immunoassay. The $\mathrm{p}-\mathrm{TDP}-43$ immunoassay was performed as previously described (33). Briefly, approximately $50 \mathrm{mg}$ postmortem tissue from the frontal cortex of patients or unaffected individuals were homogenized in cold RIPA buffer $(25 \mathrm{mM}$ Tris$\mathrm{HCl}, \mathrm{pH}$ 7.6, $150 \mathrm{mM} \mathrm{NaCl}, 1 \%$ sodium deoxycholate, 1\% Nonidet $\mathrm{P}-40,0.1 \%$ sodium dodecyl sulfate, and protease and phosphatase inhibitors) and sonicated on ice. Homogenates were centrifuged at $100,000 \mathrm{~g}$ for 30 minutes at $4^{\circ} \mathrm{C}$. The supernatant was collected, and the pellet was resuspended in RIPA buffer, sonicated and centrifuged again to prevent carryover of soluble material. The RIPA-insoluble pellet was extracted using $7 \mathrm{M}$ urea buffer and then sonicated and centrifuged at $100,000 \mathrm{~g}$ for 30 minutes at $22^{\circ} \mathrm{C}$. The protein concentration of the urea-soluble supernatant was determined by Bradford assay. p-TDP-43 levels were determined using a sandwich Meso Scale Discovery (MSD) immunoassay. The capture antibody was a mouse monoclonal antibody that detects TDP- 43 phosphorylated at serines 409/410 (1:500, no. CAC-TIP-PTD-M01, Cosmo Bio USA), and the detection antibody was a sulfo-tagged rabbit polyclonal C-terminal TDP-43 antibody (2 $\mu \mathrm{g} / \mathrm{mL}, 12892-1-A P$, Proteintech). Urea-soluble fractions were diluted in TBS to $35 \mu \mathrm{g}$ protein per well and tested in duplicate wells. Response values corresponding to the intensity of emitted light upon electrochemical stimulation of the assay plate using the MSD QUICKPLEX SQ120 were acquired.

Statistics. Comparisons of truncated STMN2 RNA in the frontal cortex between the separate non-FTLD-TDP groups of controls and patients with PSP and various groups of patients with FTLD-TDP (all FTLD-TDP patients as well as 4 different subgroups according to the TDP-43 subtype) were made using single-variable (i.e., unadjusted) and multivariable linear regression models. Multivariable models were adjusted for age at death, sex, and RIN, and truncated STMN2 RNA in the frontal cortex was examined on the base 2 logarithmic scale, owing to its skewed distribution. Regression coefficients (referred to as $\beta$ ) and 95\% CIs were estimated and interpreted as the difference in mean truncated STMN2 RNA levels in the frontal cortex (on the base 2 logarithmic scale) between the given group of FTLD-TDP patients and the given reference group (cognitively normal controls or patients with PSP). Additionally, to further assess the ability of truncated STMN2 RNA in the frontal cortex to discriminate between FTLD-TDP and non-FTLD-TDP individuals (again, separately for cognitively normal controls and patients with PSP), we estimated the area under the ROC curve (AUC); an AUC of 0.5 corresponded to the predictive ability equal to that of chance, whereas an AUC of 1.0 indicated perfect predictive ability. Bonferroni's correction was applied to adjust for the 5 different statistical tests that were performed for the separate FTLD-TDP versus control and FTLDTDP versus PSP analyses, after which $P$ values of less than 0.01 were considered statistically significant.

In a secondary analysis, we compared truncated STMN2 RNA levels between controls and patients with PSP and made pairwise comparisons of full-length STMN2 RNA and p-TDP-43 (both on the base 2 logarithmic scale) between controls, patients with PSP, and patients with FTLD-TDP using the aforementioned linear regression analyses.

In the subgroup of 238 patients with disease, associations of 5 different variables ( $\mathrm{p}$-TDP-43 levels, TDP-43 subtype, age at onset, survival after onset, and sex) with both truncated and full-length STMN2 RNA levels were evaluated using single-variable and multivariable linear regression models, where truncated and full-length STMN2 RNA levels in the frontal cortex were assessed on the base 2 logarithmic scale, and multivariable models were adjusted for predefined, potentially confounding variables. The multivariable model assessing the p-TDP-43 response in frontal cortex was adjusted for age at death, sex, and TDP-43 subtype. The multivariable model assessing TDP-43 subtype was adjusted for sex and age at death. The multivariable model assessing age at onset was adjusted for sex and TDP-43 subtype. The multivariable model assessing survival after onset was adjusted for age at onset, sex, and TDP-43 subtype. Finally, the multivariable model assessing sex was adjusted for age at death, TDP-43 subtype, and genotype. Regression coefficients and 95\% CIs were estimated and are interpreted as the change in the mean level of truncated or fulllength STMN2 RNA in the frontal cortex (on the base 2 logarithmic scale) corresponding to the presence of the given characteristic (categorical variables) or a specified increase (continuous variables). The p-TDP-43 response in the frontal cortex was examined on the base 2 logarithmic scale in linear regression analysis because of its skewed distribution. In order to adjust for the 5 different variables that were assessed for association with the separate truncated and full-length STMN2 RNA outcomes, we used Bonferroni's correction for multiple testing, after which $P$ values of less than 0.01 were considered statistically significant. All statistical tests were 2 sided. All statistical analyses described above were performed using R Statistical Software, version 3.6.2 (R Foundation for Statistical Computing).

Statistical analyses for iPSC studies and qRT-PCR of RNA extracted from human tissues were performed using GraphPad Prism 8 (GraphPad Software), in which 1-way ANOVA with Bonferroni's post hoc test was performed for multiple group comparisons, and an unpaired Student $t$ test was performed when only 2 groups were compared. The statistical test and number of independent experiments used for each analysis are indicated in the figure legends.

Study approval. The NYGC ALS Consortium samples presented in this work were acquired through various IRB protocols from member sites and the Target ALS postmortem tissue core and transferred to the NYGC in accordance with all applicable foreign, domestic, federal, state, and local laws and regulations for processing, sequencing, and analyses.

Postmortem brain tissues from patients with FTLD-TDP or PSP and from cognitively normal individuals were obtained from the Mayo Clinic Florida Brain Bank. Diagnosis was independently ascertained by trained neurologists and neuropathologists upon neurological and pathological examinations, respectively. Written informed consent was given by all participants or authorized family members, and all protocols were approved by the IRB and ethics committee of the Mayo Clinic. A summary of the patients' characteristics is provided in Table 1. 
Sample size was determined by tissue availability and the quality of RNA obtained from such samples, as described below.

Data and code availability. RNA-Seq data generated through the NYGC ALS Consortium in this study can be accessed via the NCBI's GEO database (GEO GSE137810, GSE124439, GSE116622, and GSE153960). All RNA-Seq data generated by the NYGC ALS Consortium are made immediately available to all members of the Consortium and with other consortia with whom we have a reciprocal sharing arrangement. To request immediate access to new and ongoing data generated by the NYGC ALS Consortium and for samples provided through the Target ALS Postmortem Core, complete a genetic data request form at ALSData@nygenome.org.

\section{Author contributions}

MP, JH, and SP share co-first authorship. The order of their names was established on the basis of their individual contributions and the importance of those contributions to this study. MP led the human brain RNA and protein studies that provided the basis of the manuscript. JH led the RNA-Seq data analyses that complemented the RNA/protein studies and increased the impact of this manuscript. SP led iPSC studies and was closely involved in the RNA/protein studies as well. MP, JH, SP, TFG, PF, and LP designed the study. MP, SP, ALB, CC, YS, MY, LMD, YC, KJW, CB, AC, KT, $\mathrm{BTS}, \mathrm{KB}, \mathrm{SC}$, and EMCF generated the iPSC lines and processed iPSC and human tissue samples for qRT-PCR and RNA-Seq. SEH and MW contributed to the experimental design for CRISPRi knockdown of TARDBP in iPSC-derived neurons. In the NYGC ALS Consortium, members contributed postmortem samples and clinical information that were curated deidentified by DK. and processed for RNA-Seq by IH, DC, and NP. NP and SF oversee Consortium resources and data distribution. DF and HP designed the methodology, reviewed sample and data quality, and coordinated NYGC ALS Consortium postmortem RNA research activity. JH, YCW, PS, ALB, MS, and TR performed RNA-Seq data analyses. MP, JMK, JS, EAT performed NanoString assays and analyzed the data. SP and TFG performed p-TDP-43 immunoassays. MP, $\mathrm{MGH}, \mathrm{MRS}, \mathrm{ALB}$, and MS performed statistical analyses. MP, JH, SP, ALB, TFG, PF, and LP wrote the manuscript. CFDC, MD, SK, JN, TL, the NYGC ALS Consortium, BO, MVB, RR, NRGR, BFB,
DSK, RCP, KAJ, and DWD provided human tissue samples as well as pathological, genetic, and clinical information. All authors read and approved the manuscript.

\section{Acknowledgments}

We thank the Target ALS Postmortem Core for providing postmortem brain samples, and we thank all the patients and their families for their contribution to this study. See Supplemental Acknowledgments for consortium details. This work was supported by the NINDS, NIH (R35NS097273, P01NS084974, R21NS084528, and R01NS088689, to LP; P01NS099114, to TFG and LP; R01AG037491, to KAJ; P50AG016574, to RCP and BFB; U01AG006786, to RCP; and U01AG045390, to BFB); the National Institute on Aging (R56AG055824, to JH and TR); the Department of Defense (ALSRP AL130125, to LP); the Mayo Clinic Foundation (to LP); the Association of Frontotemporal Dementia (AFTD) (to LP); the Amyotrophic Lateral Sclerosis Association (to LP and MP); the Robert Packard Center for ALS Research at Johns Hopkins (to LP); Target ALS (to TFG and LP); the Canadian Institute of Health Research (to SP); the UK Medical Research Council (to $\mathrm{PF}$ and EMCF); the Motor Neuron Disease Association (to PF); the Rosetrees Foundation (to PF and EMCF); the National Institute for Health Research (NIHR) University College London Hospitals (UCLH) Biomedical Research Centre (to PF and ALB); Alzheimer's Research UK (to TL); and the UK Dementia Research Institute, which receives its funding from DRI Ltd., supported by the UK Medical Research Council, the Alzheimer's Society, and Alzheimer's Research UK (to BTS and SC). This work was also supported in part by the Intramural Research Program, NINDS, NIH (to MW). All NYGC ALS Consortium activities are supported by the ALS Association (15-LGCA-234) and the Tow Foundation.

Address correspondence to: Leonard Petrucelli, Department of Research, Neuroscience, Mayo Clinic College of Medicine, 4500 San Pablo Road, Jacksonville, Florida 32224, USA. Phone: 904.953.2855; Email: petrucelli.leonard@mayo.edu. Or to: Pietro Fratta, Department of Neuromuscular Diseases, UCL Queen Square Institute of Neurology, Queen Square, London, WC1N 3BG, United Kingdom. Phone: 44.0.2034484112; Email:p.fratta@ucl.ac.uk.
1. Ling SC, Polymenidou M, Cleveland DW. Converging mechanisms in ALS and FTD: disrupted RNA and protein homeostasis. Neuron. 2013;79(3):416-438.

2. Polymenidou M, et al. Long pre-mRNA depletion and RNA missplicing contribute to neuronal vulnerability from loss of TDP-43. Nat Neurosci. 2011;14(4):459-468.

3. Tollervey JR, et al. Characterizing the RNA targets and position-dependent splicing regulation by TDP-43. Nat Neurosci. 2011;14(4):452-458.

4. Ling JP, Pletnikova O, Troncoso JC, Wong PC. TDP-43 repression of nonconserved cryptic exons is compromised in ALS-FTD. Science. 2015;349(6248):650-655.

5. Jeong YH, et al. Tdp-43 cryptic exons are highly variable between cell types. Mol Neurodegener. 2017;12(1):13.

6. Humphrey J, Emmett W, Fratta P, Isaacs AM, Plagnol V. Quantitative analysis of cryptic splicing associated with TDP-43 depletion. BMC Med Genomics. 2017;10(1):38.

7. Klim JR, et al. ALS-implicated protein TDP43 sustains levels of STMN2, a mediator of motor neuron growth and repair. Nat Neurosci. 2019;22(2):167-179.

8. Melamed Z, et al. Premature polyadenylation-mediated loss of stathmin-2 is a hallmark of TDP-43-dependent neurodegeneration. Nat Neurosci. 2019;22(2):180-190.

9. Shin JE, Geisler S, DiAntonio A. Dynamic regulation of SCG10 in regenerating axons after injury. Exp Neurol. 2014;252:1-11.

10. Neumann M, Mackenzie IRA. Review: Neuropathology of non-tau frontotemporal lobar degeneration. Neuropathol Appl Neurobiol. 2019;45(1):19-40.

11. Sreedharan J, et al. TDP-43 mutations in familial and sporadic amyotrophic lateral sclerosis. $\mathrm{Sci}$ ence. 2008;319(5870):1668-1672.

12. Borroni B, et al. Mutation within TARDBP leads to frontotemporal dementia without motor neuron disease. Hum Mutat. 2009;30(11):E974-83.

13. Mackenzie IR, et al. Dipeptide repeat protein pathology in C9ORF72 mutation cases: clinico-pathological correlations. Acta Neuropathol. 2013;126(6):859-879.

14. Neumann M, et al. Ubiquitinated TDP-43 in frontotemporal lobar degeneration and amyotrophic lateral sclerosis. Science. 2006;314(5796):130-133.

15. Sampathu DM, et al. Pathological heterogeneity of frontotemporal lobar degeneration with ubiquitin-positive inclusions delineated by ubiquitin immunohistochemistry and novel monoclonal antibodies. Am J Pathol. 2006;169(4):1343-1352.

16. Davidson $Y$, et al. Ubiquitinated pathological lesions in frontotemporal lobar degeneration contain the TAR DNA-binding protein, TDP-43. Acta Neuropathol. 2007;113(5):521-533.

17. Arai T, et al. TDP-43 is a component of ubiquitin-positive tau-negative inclusions in fronto- 
temporal lobar degeneration and amyotrophic lateral sclerosis. Biochem Biophys Res Commun. 2006;351(3):602-611.

18. Hatanpaa KJ, et al. TAR DNA-binding protein 43 immunohistochemistry reveals extensive neuritic pathology in FTLD-U: a midwest-southwest consortium for FTLD study. J Neuropathol Exp Neurol. 2008;67(4):271-279.

19. Josephs KA, Stroh A, Dugger B, Dickson DW. Evaluation of subcortical pathology and clinical correlations in FTLD-U subtypes. Acta Neuropathol.2009;118(3):349-358.

20. Mackenzie IR, et al. A harmonized classification system for FTLD-TDP pathology. Acta Neuropathol. 2011;122(1):111-113.

21. Lee EB, et al. Expansion of the classification of FTLD-TDP: distinct pathology associated with rapidly progressive frontotemporal degeneration. Acta Neuropathol. 2017;134(1):65-78.

22. Mackenzie IR, et al. Heterogeneity of ubiquitin pathology in frontotemporal lobar degeneration: classification and relation to clinical phenotype. Acta Neuropathol. 2006;112(5):539-549.

23. Tan RH, et al. Classification of FTLD-TDP cases into pathological subtypes using antibodies against phosphorylated and non-phosphorylated TDP43. Acta Neuropathol Commun. 2013;1:33.

24. Josephs KA, et al. Neuropathological background of phenotypical variability in frontotemporal dementia. Acta Neuropathol. 2011;122(2):137-153.

25. Josephs KA, et al. Neuropathologic features of frontotemporal lobar degeneration with ubiquitin-positive inclusions with progranulin gene (PGRN) mutations. J Neuropathol Exp Neurol. 2007;66(2):142-151.

26. Mackenzie IR, et al. The neuropathology of frontotemporal lobar degeneration caused by mutations in the progranulin gene. Brain. 2006;129(Pt 11):3081-3090.

27. Feneberg E, Gray E, Ansorge O, Talbot K, Turner MR. Towards a TDP-43-based biomarker for ALS and FTLD. Mol Neurobiol. 2018;55(10):7789-7801.

28. Nana AL, et al. Neurons selectively targeted in frontotemporal dementia reveal early stage TDP-43 pathobiology. Acta Neuropathol. 2019;137(1):27-46

29. Koga S, et al. Corticobasal degeneration with TDP-43 pathology presenting with progressive supranuclear palsy syndrome: a distinct clinicopathologic subtype. Acta Neuropathol. 2018;136(3):389-404.

30. Tian R, et al. CRISPR interference-based platform for multimodal genetic screens in human iPSCderived neurons. Neuron. 2019;104(2):239-255.e12.

31. Fratta $P$, et al. Mice with endogenous TDP- 43 mutations exhibit gain of splicing function and characteristics of amyotrophic lateral sclerosis. EMBO J. 2018;37(11):e98684.

32. Jiang J, et al. Gain of toxicity from ALS/ FTD-linked repeat expansions in C9ORF72 is alleviated by antisense oligonucleotides targeting GGGGCC-containing RNAs. Neuron. 2016;90(3):535-550.

33. Prudencio M, et al. Repetitive element transcripts are elevated in the brain of C9orf72 ALS/FTLD patients. Hum Mol Genet. 2017;26(17):3421-3431.

34. Schludi MH, et al. Spinal poly-GA inclusions in a C9orf72 mouse model trigger motor deficits and inflammation without neuron loss. Acta Neuro- pathol. 2017;134(2):241-254.

35. Bang J, Spina S, Miller BL. Frontotemporal dementia. Lancet. 2015;386(10004):1672-1682.

36. Wang $\mathrm{Q}$, et al. The landscape of multiscale transcriptomic networks and key regulators in Parkinson's disease. Nat Commun. 2019;10(1):5234.

37. Amador-Ortiz C, et al. TDP-43 immunoreactivity in hippocampal sclerosis and Alzheimer's disease. Ann Neurol. 2007;61(5):435-445.

38. Arai T, et al. Phosphorylated TDP-43 in Alzheimer's disease and dementia with Lewy bodies. Acta Neuropathol. 2009;117(2):125-136.

39. Higashi S, et al. Concurrence of TDP-43, tau and alpha-synuclein pathology in brains of Alzheimer's disease and dementia with Lewy bodies. Brain Res. 2007;1184:284-294.

40. Hu WT, et al. Temporal lobar predominance of TDP43 neuronal cytoplasmic inclusions in Alzheimer disease. Acta Neuropathol. 2008;116(2):215-220.

41. Josephs KA, et al. Abnormal TDP-43 immunoreactivity in AD modifies clinicopathologic and radiologic phenotype. Neurology. 2008;70(19 Pt 2):1850-1857.

42. Kadokura A, et al. Phosphorylation-dependent TDP-43 antibody detects intraneuronal dot-like structures showing morphological characters of granulovacuolar degeneration. Neurosci Lett. 2009;463(1):87-92.

43. Kadokura A, Yamazaki T, Lemere CA, Takatama M, Okamoto K. Regional distribution of TDP-43 inclusions in Alzheimer disease (AD) brains: their relation to $\mathrm{AD}$ common pathology. Neuropathology. 2009;29(5):566-573.

44. Uryu K, et al. Concomitant TAR-DNA-binding protein 43 pathology is present in Alzheimer disease and corticobasal degeneration but not in other tauopathies. J Neuropathol Exp Neurol. 2008;67(6):555-564.

45. Josephs KA, et al. Rates of hippocampal atrophy and presence of post-mortem TDP-43 in patients with Alzheimer's disease: a longitudinal retrospective study. Lancet Neurol. 2017;16(11):917-924.

46. Josephs KA, et al. TAR DNA-binding protein 43 and pathological subtype of Alzheimer's disease impact clinical features. Ann Neurol. 2015;78(5):697-709.

47. Chen S, Zhou Y, Chen Y, Gu J. fastp: an ultra-fast all-in-one FASTQ preprocessor. Bioinformatics. 2018;34(17):i884-i90.

48. Dobin A, et al. STAR: ultrafast universal RNA-sec aligner. Bioinformatics. 2013;29(1):15-21.

49. Liao Y, Smyth GK, Shi W. featureCounts: an efficient general purpose program for assigning sequence reads to genomic features. Bioinformatics 2014;30(7):923-930.

50. Robinson MD, McCarthy DJ, Smyth GK. edgeR: a Bioconductor package for differential expression analysis of digital gene expression data. Bioinformatics. 2010;26(1):139-140.

51. Katz Y, Wang ET, Airoldi EM, Burge CB. Analysis and design of RNA sequencing experiments for identifying isoform regulation. Nat Methods. 2010;7(12):1009-1015.

52. Tam OH, et al. Postmortem cortex samples identify distinct molecular subtypes of ALS: retrotransposon activation, oxidative stress, and activated glia. Cell Rep. 2019;29(5):1164-1177.

53. Di Tommaso P, Chatzou M, Floden EW, Barja PP, Palumbo E, Notredame C. Nextflow enables reproducible computational workflows. Nat Biotechnol. 2017;35(4):316-319.

54. Bolger AM, Lohse M, Usadel B. Trimmomatic: a flexible trimmer for Illumina sequence data. Bioinformatics. 2014;30(15):2114-2120.

55. Frankish A, et al. GENCODE reference annotation for the human and mouse genomes. Nucleic Acids Res. 2019;47(D1):D766-D73.

56. Li B, Dewey CN. RSEM: accurate transcript quantification from RNA-Seq data with or without a reference genome. BMC Bioinformatics. 2011;12:323.

57. Li H, et al. The Sequence Alignment/ Map format and SAMtools. Bioinformatics. 2009;25(16):2078-2079.

58. [No authors listed]. Picard Toolkit. Broad Institute. http://broadinstitute.github.io/picard/. Accessed September 3, 2020.

59. Ewels P, Magnusson M, Lundin S, Kaller M. MultiQC: summarize analysis results for multiple tools and samples in a single report. Bioinformatics. 2016;32(19):3047-3048.

60. R Core Team. R: A language and environment for statistical computing. R Foundation for Statistica Computing, Vienna, Austria. http://www.R-project.org/. Accessed September 3, 2020.

61. Wickham H. tidyverse: Easily Install and Load the 'Tidyverse'. https://cran.r-project.org/web/packages/tidyverse/index.html. Published November 21, 2019. Accessed September 3, 2020.

62. Kassambara A. ggpubr: 'ggplot2' Based Publication Ready Plots. Github. https://github.com/kassambara/ggpubr. Accessed Septmeber 3, 2020.

63. Pedersen TL. patchwork: the composer of plots. R Project. https://cran.r-project.org/web/packages/patchwork/index.html. Published June 22, 2020. Accessed September 3, 2020.

64. Aguet F, et al. The GTEx Consortium atlas of genetic regulatory effects across human tissues. bioRxiv. https://doi.org/10.1101/787903. Published October 3, 2019. Accessed September 3, 2020.

65. Schroeder A, et al. The RIN: an RNA integrity number for assigning integrity values to RNA measurements. BMC Mol Biol. 2006;7:3.

66. Jaffe AE, et al. qSVA framework for RNA quality correction in differential expression analysis. Proc Natl Acad Sci U S A. 2017;114(27):7130-7135.

67. Koster J, Rahmann S. Snakemake-a scalable bioinformatics workflow engine. Bioinformatics. 2012;28(19):2520-2522.

68. Humphrey J. STMN2 splicing pipeline. https:// github.com/RajLabMSSM/STMN2-splicing. Accessed September 3, 2020.

69. Feng YY, , et al. RegTools: Integrated analysis of genomic and transcriptomic data for discovery of splicing variants in cancer. bioRxiv. https://doi. org/10.1101/436634. Published November 25, 2018. Accessed September 3, 2020.

70. Li YI, et al. Annotation-free quantification of RNA splicing using LeafCutter. Nat Genet. 2018;50(1):151-158.

71. Jackson JL, et al. Elevated methylation levels, reduced expression levels, and frequent contrac tions in a clinical cohort of C9orf72 expansion carriers. Mol Neurodegener. 2020;15(1):7.

72. Dafinca R, et al. Impairment of mitochondrial calcium buffering links mMutations in C9ORF72 and TARDBP in iPS-derived motor neurons from patients with ALS/FTD. Stem Cell Reports. 2020;14(5):892-908 